\title{
Monitoreo Comunitario y Participativo de los Manglares bajo Acuerdos de Uso y Custodia de Manglar en Ecuador
}

\section{Mangroves Community and Participatory Monitoring under Agreements for Mangrove Sustainable Use and Custody in Ecuador}

INFORMACIÓN DEL ARTÍCULO

Fecha de recepción: 21 de enero de 2020.

Fecha de aceptación: 6 de marzo de 2020
${ }^{1}$ Asesora Interna Universidad Particular de Loja. Cooperación Técnica Alemana (UTPL-GIZ), Loja, Ecuador.

E-mail: irma.jurrius@giz.de Código ORCID:

https://orcid.org/0000-0002-5220-5636

${ }^{2}$ Universidad Técnica Particular de Loja (UTPL), Loja, Ecuador.

E-mail: fvlopezx@utpl.edu.ec

Código ORCID:

https://orcid.org/0000-0002-9946-0992

CITACIÓN: Jurrius, I. \& López Rodríguez, F. (2020). Monitoreo Comunitario y Participativo de los Manglares bajo. Acuerdos de Uso y Custodia de Manglar en Ecuador Investigatio, 14, 27-37.

doi:10.31095/investigatio.2020.14.3

ENLACE DOI:

http://dx.doi.org/10.31095/investigatio. 2020.14 .3

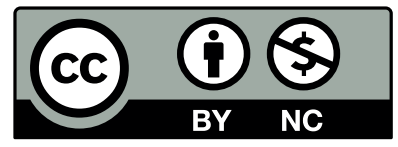

Irma María Jurrius ${ }^{1}$, Fausto Vinicio López Rodríguez ${ }^{2}$

\section{Resumen}

El marco normativo de los Acuerdos de Uso Sustentable y Custodia de Manglar (AUSCM) plantea a los informes semestrales como el instrumento para informar al Ministerio del Ambiente (MAE) sobre la gestión de un área de custodia, dejando la responsabilidad de recolectar la información y formular dichos informes en manos de la organización con la cual se firma el Acuerdo. En este estudio se analizó, con elementos de la normativa y la literatura considerar estos informes dentro del concepto de Monitoreo Participativo y Comunitario de los recursos naturales, basado en la experiencia de 10 organizaciones de la Provincia de El Oro bajo AUSCM. Se concluyó que, se puede considerar un monitoreo participativo y comunitario, y fortalecerlo incluyendo indicadores sobre el estado de los manglares bajo AUSCM, entre otros. Este monitoreo podría servir como insumo de información relevante para la toma de decisiones sobre la conservación del manglar.

\section{Palabras Clave: \\ Monitoreo Participativo y Comunitario, Informes semestrales, Manglares, AUCM.}

Clasificación JEL: Q10, Q24.

\begin{abstract}
The regulations on the sustainable use and custody agreements of mangrove forests (SUCAM) proposes the elaboration of semiannual reports as an instrument to inform the MAE about management of the mangroves, leaving the responsibility of collecting the information and formulating or these reports in the hands of the fishermen organizations with whom these mangrove agreements are signed. This study analyzed; based on the experience of 10 organizations of the Province of El Oro, elements of the regulations, and literature, if this way of reporting can be contemplated within the framework of "Participatory and Community Monitoring of natural resources". It was concluded that it can be considered as participatory and community monitoring, and if it is complemented by including indicators on the status of mangroves under SUCAM, among others, this monitoring could serve as an input of relevant information for decision making processes on mangrove conservation.
\end{abstract}

Keywords:

Participatory and community monitoring, bianmual reports, mangroves, AMSUC. JEL Classification: Q10, Q24. 


\section{Introducción}

A nivel mundial, los manglares se encuentran bajo mucha presión humana, lo cual ha puesto en riesgo los servicios que brindan a la población costera (Lopez-Rodriguez, 2018; Alongi, 2002; McIvor et al., 2015; Menéndez, 2013). Una estrategia para ampliar la base de conocimiento sobre el deterioro de los ecosistemas y generar insumos para la toma de decisiones para políticas y normativa es el monitoreo (Stelzenmuller, 2013; PNUD- IAVH, 2017; Capote-Fuentes y Levins, 2006). A nivel de Latino América existen varias iniciativas de monitoreo de los manglares promovidas, principalmente, por entes del Estado, como es el caso de México (CONABIO, 2005), Cuba (Menéndez, 2012) y Colombia (INVEMAR, 2012).

Abbot y Gujit (1998) han identificado a los siguientes actores que pueden ser responsables para el monitoreo: investigadores, entes de Estado, usuarios de los recursos naturales, ONG, etc. En esta investigación nos enfocamos en el monitoreo participativo y comunitario, que según Abbot y Guijt (1998) y Evans (2008) se define como el monitoreo que se desarrolla entre varios actores, incluyendo los usuarios, para lograr un monitoreo eficiente, efectivo y más social. Evans (2016) presenta una caracterización del nivel de participación de los usuarios en el monitoreo participativo que va de menor a mayor grado 1) Participación nominal 2) participación pasiva 3) participación activa 4) participación consultiva y 5) participación de empoderamiento.
Así mismo, diferencian otra forma de categorizar el monitoreo de los recursos naturales, basado en el objeto del monitoreo. De la revisión y análisis de documentos de Abbot y Guijt (1998), Stelzenmuller (2013), Evans (2018), Fröde y Masara (2007) se identificaron los siguientes temas que se abordan en los procesos de monitoreo participativo de los recursos naturales: 1) estado de los recursos naturales y su ecosistema 2) estado de especies útiles 3) amenazas a los recursos naturales 4) acciones de manejo 5) cumplimiento de la normativa 6) análisis de efectividad de un proyecto.

Se puede diferenciar el monitoreo también por las metodologías que se usan para recolectar los datos. Hay una variedad de publicaciones que presentan metodologías participativas (Bautista et al., 2004; Gallego, 2017) dentro de la cual se encuentran: mediciones en campo y laboratorios, observación en campo, llenado de fichas, SIG, revisión documental, talleres participativos, entrevistas y encuestas.

En Ecuador, todos los bosques de manglar son bienes del Estado (COA, 2017) siendo el MAE la institución responsable de su manejo. Los AUSCM son la herramienta de gestión contemplada en marco legal ecuatoriano (COA, 2017), bajo los cuales se entregan en custodia bosques del manglar a usuarios ancestrales asentados a lo largo del perfil costanero. Los AUSCM garantizan a los custodios el acceso exclusivo a las áreas de manglar con el derecho de aprovechar sustentablemente los recursos bioacuáticos, pero a su vez tienen la obligación de 
custodiar el manglar y reportar los avances de su gestión al MAE (Acuerdo Ministerial $\left.N^{\circ} 129,2010\right)$.

El Plan Nacional para la Conservación de los Manglares del Ecuador Continental elevó la importancia del monitoreo comunitario, al tener dentro de sus metas 1) promover la investigación científica $\mathrm{y}$ aplicada enfocado en el manglar, sus servicios ecosistémicos, la vulnerabilidad del manglar y las comunidades que depende de él; 2) potenciar el monitoreo participativo de los recursos pesqueros y 3) promover acciones de control y vigilancia participativa con fines de identificar impactos de origen humano al ecosistema manglar (MAE, 2019).

En esta investigación se analiza la manera en la que las organizaciones con AUSCM en la provincia de El Oro informan sobre la gestión y estado de los manglares bajo su custodia, y plantea recomendaciones para mejorar su efectividad. Al final se espera contestar la siguiente pregunta: ¿la manera en que las organizaciones recolectan datos y reportan sobre el manglar bajo su custodia se enmarca en el concepto conocido a nivel mundial como el "monitoreo participativo y comunitario de los recursos naturales"?

\section{Métodos y área de estudio}

Para el análisis de la forma en que las organizaciones reportan sobre los manglares bajo su custodia, se realizó un estudio exploratorio, analizando los informes de 10 organizaciones de pescadores de la provincia de El Oro con AUSCM a las cuales la Universidad
Técnica Particular de Loja (UTPL) brinda asistencia técnica. Los informes semestrales fueron elaborados en el mes de enero 2019, entre las organizaciones, docentes, estudiantes de la UTPL y la Cooperación Técnica Alemana (GIZ).

Se partió de un análisis documental de la normativa de los AUSCM para conocer los temas y la metodología propuesta para la forma en que las organizaciones deben informar al MAE, el cual se comparó con la literatura sobre el monitoreo de los recursos naturales. Con base en este análisis se propusieron ocho categorías que se consideran claves para el monitoreo de los manglares bajo custodia.

Posteriormente se calificaron los 10 informes semestrales de las organizaciones seleccionados y se analizó el número de veces que estos informes abordan elementos de las ocho categorías identificadas. Para la calificación se usaron los siguientes criterios:

- El informe no contiene ninguna cita sobre los elementos objeto de esta categoría: 0 puntos

- Uno o dos citas: 1 punto

- Tres o cuatro menciones: 2 puntos

- Más de cinco menciones: 3 puntos.

Para conocer si la forma de recolectar la información y de elaborar los informes semestrales se enmarca en el concepto de monitoreo participativo y comunitario, se realizó un análisis de la normativa de los AUSCM, el cual se comparó con el concepto de monitoreo participativo y comunitario de los recursos naturales presente en la literatura y con la 
metodología de trabajo empleada en Ecuador por UTPL-GIZ.

\section{Resultados}

Del análisis de la normativa se evidenció que el Acuerdo Ministerial Nro. 129 plantea como obligación de las organizaciones que firman un Acuerdo, "Informar semestralmente sobre el estado del uso sustentable y custodia del manglar y los avances en el cumplimiento del Plan de Manejo" (Acuerdo Ministerial Nro. 129, 2010), siendo así los informes semestrales la metodología para el monitoreo por parte de las organizaciones con AUSCM.

Para este fin, en el anexo 4 del Acuerdo Ministerial No. 144 se presenta un formato que aborda una matriz de cumplimiento del Plan de Manejo en la cual se coloca una descripción de las actividades cumplidas y las evidencias o medios de verificación respectivas. Es importante destacar que el formato de los informes semestrales no tiene espacios para incluir información sobre "el estado del uso sustentable y custodia del manglar". Es por esta razón que se analizó a mayor profundidad los artículos del Acuerdo Ministerial Nro. 129 en el cual se detallan los derechos y obligaciones que contemplan los AUSCM para poder identificar qué temas, mediante normativa podrían ser objeto de un monitoreo participativo de los AUSCM, información que se encuentra en la Tabla 1.

Tabla 1.

Temas objeto de monitoreo planteados en el Acuerdo Ministerial Nro. 129

\begin{tabular}{|c|c|}
\hline \multicolumn{2}{|r|}{ Temas objeto de monitoreo planteado en la normativa vigente de AUSCM (A.M.129) } \\
\hline $\begin{array}{l}\text { Art } 2 \\
\text { (Derecho) }\end{array}$ & $\begin{array}{l}\text { Uso sustentable del manglar bajo custodia dando un aprovechamiento no destructivo de } \\
\text { los recursos naturales de este ecosistema (Pesca extractiva de peces e invertebrados, cría, } \\
\text { engorde o cultivo de fauna nativa, reforestación del manglar, turismo ecológico, } \\
\text { conservación y protección, educación e investigación científica) }\end{array}$ \\
\hline $\begin{array}{l}\text { Art } 3 \\
\text { (Deber) }\end{array}$ & $\begin{array}{l}\text { Contar con un Plan de Manejo con Programa de aprovechamiento sustentable, Control y } \\
\text { Vigilancia y Monitoreo/evaluación }\end{array}$ \\
\hline $\begin{array}{l}\text { Art } 4 \\
\text { (Deber) }\end{array}$ & Contar con un reglamento interno con medidas de manejo y sanciones \\
\hline $\begin{array}{l}\text { Art } 5 \\
\text { (Deber) }\end{array}$ & Contar con un convenio de asistencia técnica para el manejo sustentable del manglar \\
\hline $\begin{array}{l}\text { Art } 8 \\
\text { (Derecho) }\end{array}$ & $\begin{array}{l}\text { Aprovechar sustentablemente y en forma exclusiva los recursos del área de manglar bajo } \\
\text { custodia, derecho que no será susceptible a cesión, fraccionamiento o enajenación }\end{array}$ \\
\hline $\begin{array}{l}\text { Art } 9 \\
\text { (Deber) }\end{array}$ & $\begin{array}{l}\text { Custodiar el manglar y denunciar inminentemente cualquier anomalía } \\
\text { Cumplir el Plan de Manejo }\end{array}$ \\
\hline & $\begin{array}{l}\text { Cumplir las disposiciones legales de los ámbitos pesquero, acuícola, turístico, ambiental } \\
\text { y uso de zonas de playa y bahía } \\
\text { Informar semestralmente sobre el estado del uso sustentable y custodia del manglar y } \\
\text { avances en el cumplimiento del Plan } \\
\text { Mantener actualizada y vigente la personería jurídica de la organización }\end{array}$ \\
\hline $\begin{array}{l}\text { Art } 12 \\
\text { (Deber) }\end{array}$ & $\begin{array}{l}\text { Entregar a tiempo los informes semestrales } \\
\text { Cumplir con el calendario o cronograma del Plan de Manejo } \\
\text { Informar oportunamente de cambios en la organización beneficiaria (Directiva, socios, } \\
\text { estatutos reglamentos) }\end{array}$ \\
\hline
\end{tabular}

Fuente: Elaboración propia 
Del análisis de los temas que forman parte del monitoreo de recursos naturales presente en la literatura y posibles temas de monitoreo que se encuentran en la normativa de los AUSCM, se generaron ocho categorías sobre las cuales se podría realizar un monitoreo participativo de

Tabla 2.

Análisis de posibles temas de monitoreo orientados al cumplimiento del Acuerdo Ministerial Nro. 129

\begin{tabular}{ll}
\hline $\begin{array}{l}\text { Categorías objeto de } \\
\text { monitoreo de los }\end{array}$ & Ejemplo de características del objeto de monitoreo \\
AUSCM & \\
\hline $\begin{array}{l}\text { Estado del ecosistema } \\
\text { manglar bioacuáticos }\end{array}$ & $\begin{array}{l}\text { Cobertura de manglar/clima/presencia de flora y fauna/enfermedad o muerte } \\
\text { de especies de flora y fauna/olores/colores del agua y sedimento }\end{array}$ \\
\hline $\begin{array}{l}\text { Uso Sustentable } \\
\text { de ecursos }\end{array}$ & $\begin{array}{l}\text { Ingresos/esfuerzo pesquero/medidas de recursos recolectados, captura por } \\
\text { Unidad de Esfuerzo (CPUE), densidad, etc. }\end{array}$ \\
\hline $\begin{array}{l}\text { Amenazas y anomalías } \\
\text { en el Manglar }\end{array}$ & $\begin{array}{l}\text { Notificaciones y denuncias sobre anomalías como contaminación/tala/muerte } \\
\text { de recursos/sobreexplotación de recursos }\end{array}$ \\
\hline $\begin{array}{l}\text { Cumplimiento del Plan } \\
\text { de Manejo }\end{array}$ & Cumplimiento de acciones propuestas en el Plan \\
\hline $\begin{array}{l}\text { Cumplimiento del } \\
\text { Reglamento Interno } \\
\text { Cumplimiento del }\end{array}$ & $\begin{array}{l}\text { Cumplimiento de las reglas de la organización en cuanto al manejo del } \\
\text { Convenio de }\end{array}$ \\
$\begin{array}{l}\text { Asistencia Técnica } \\
\text { Cumplimiento de la } \\
\text { normativa }\end{array}$ & Actualización del convenio, Cumplimiento del convenio \\
\hline $\begin{array}{l}\text { Desarrollo } \\
\text { organizacional }\end{array}$ & Cumplimiento de normativa ambiental/pesca/turismo, etc \\
\hline
\end{tabular}

Fuente: Elaboración propia.

Tabla 3.

Presencia de información de monitoreo de AUSCM según 8 categorías

\begin{tabular}{|c|c|c|c|c|c|c|c|c|}
\hline $\begin{array}{c}\text { Objetos de monitoreo } \\
\text { reportados en los } \\
\text { informes semestrales } \\
\text { del } 2018 \\
\text { Organizaciones }\end{array}$ & $\begin{array}{l}\text { Estado } \\
\text { del } \\
\text { ecosis } \\
\text { tema } \\
\text { manglar }\end{array}$ & $\begin{array}{c}\text { Uso } \\
\text { sustenta } \\
\text { ble de } \\
\text { recursos } \\
\text { bioacuá } \\
\text { ticos }\end{array}$ & $\begin{array}{c}\text { Amenazas } \\
y \\
\text { anomalias } \\
\text { en el } \\
\text { manglar }\end{array}$ & $\begin{array}{c}\text { Cumpli } \\
\text { miento del } \\
\text { Plan de } \\
\text { Manejo }\end{array}$ & $\begin{array}{c}\text { Cumpli } \\
\text { miento de } \\
\text { Reglamen } \\
\text { to Interno }\end{array}$ & $\begin{array}{l}\text { Cumpli } \\
\text { miento de } \\
\text { Norma } \\
\text { tiva }\end{array}$ & $\begin{array}{c}\text { Cumpli } \\
\text { miento } \\
\text { convenio } \\
\text { de } \\
\text { Asistencia } \\
\text { Técnica }\end{array}$ & $\begin{array}{c}\text { Desarrollo } \\
\text { organiza } \\
\text { cional }\end{array}$ \\
\hline Isla Bellavista & 1 & 2 & 1 & 3 & 0 & 1 & 1 & 3 \\
\hline Costa Rica & 1 & 2 & 1 & 3 & 0 & 1 & 1 & 3 \\
\hline 24 de Octubre & 1 & 2 & 1 & 3 & 0 & 0 & 1 & 3 \\
\hline 19 de Octubre & 1 & 2 & 1 & 3 & 0 & 0 & 1 & 3 \\
\hline Amor y Esperanza & 0 & 2 & 1 & 3 & 0 & 1 & 1 & 3 \\
\hline Puerto Grande & 1 & 2 & 2 & 3 & 0 & 0 & 1 & 3 \\
\hline 17 de Enero & 0 & 2 & 1 & 3 & 0 & 0 & 1 & 3 \\
\hline Vikingos del Mar & 1 & 2 & 2 & 3 & 0 & 1 & 1 & 3 \\
\hline 11 de enero & 1 & 2 & 2 & 3 & 0 & 1 & 1 & 3 \\
\hline Estero porteño & 2 & 2 & 2 & 3 & 0 & 1 & 1 & 3 \\
\hline Total & 9 & 20 & 14 & 30 & 0 & 6 & 10 & 30 \\
\hline
\end{tabular}

Fuente: Elaboración propia. 
con estas 8 categorías en los 10 informes semestrales objeto del estudio.

Los informes semestrales analizados abordan, principalmente, el cumplimiento del Plan de Manejo, tal como se solicita en el formato del Acuerdo Ministerial No 144 , con una ponderación total de 30 (sumatoria de todas las organizaciones), puntaje máximo en este análisis. En la categoría Desarrollo Organizacional alcanzaron un puntaje de 30 lo cual demuestra que las organizaciones son activas y buscan mejorar su desempeño en la gestión de los manglares. Como los planes de manejo contemplan en su Programa de Control y Vigilancia la custodia del área de manglar, se llegó a tener un puntaje de 15 para esta categoría. Lo mismo en cuanto al Programa de Aprovechamiento y Uso Sustentable, el cual logró un puntaje de 20. El programa de Monitoreo y Seguimiento plantea la gestión de convenios de asistencia técnica, por ende, todas las organizaciones hicieron mención, por lo menos 1 vez, a estos convenios, pero no todos explican con detalle el apoyo que recibieron de su ente de asistencia técnica. Las demás categorías: Estado del manglar y Cumplimiento de normativa y Reglamento Interno tuvieron puntajes menores a 10 , siendo entonces los temas que reciben menor atención en los informes.

En cuanto a la pregunta si el monitoreo de los manglares bajo AUSCM es comunitario y participativo en su naturaleza, es importante anotar que la elaboración de informes semestrales está contemplada en el Art 9 del Acuerdo Ministerial Nro. 129 como deber de las organizaciones con AUSCM, por lo que por normativa si se enmarca en el concepto del monitoreo participativo y comunitario. En la práctica ha sido difícil para las organizaciones elaborar estos informes semestrales, ya que no cuentan con una cultura de registrar lo que observan durante sus recorridos de control y vigilancia, recolectar evidencias de las actividades realizadas en el marco de la gestión del manglar, y de archivar información de manera ordenada para su uso posterior en los informes semestrales (UTPL, 2017).

De las diez organizaciones objeto de este estudio, hasta el 2017, tres no entregaron oportunamente sus informes, $\mathrm{y}$ todos unificaron dos o tres semestres en un solo informe y entregaron de manera atrasada sus informes (UTPL, 2017). Siete de las organizaciones tuvieron una calificación "no satisfactoria" por parte del MAE-SGMC por: falta de medios de verificación adecuados; cumplimiento parcial de actividades; incumplimiento de los tiempos y presentación de informes unificados correspondientes a varios semestres (UTPL, 2017). En el 2017 y 2018, años en los cuales la UTPL-GIZ empezaron a brindar asistencia técnica en la elaboración de informes semestrales, estas organizaciones cumplieron con la entrega oportuna de estos informes, aunque sigue siendo deficiente la presentación de evidencias de cumplimiento de sus Planes de Manejo.

La UTPL-GIZ trabajó los informes del 2018 por medio de talleres participativos y la revisión documental de evidencias de la gestión (informes, listas 
participantes, facturas, fotos, invitaciones, etc.). Se usaron para ello técnicas participativas de recolección de la información, como la "línea del tiempo", para identificar las actividades realizadas y "lluvia de ideas" sobre evidencias de estas actividades. Se usaron papelotes para visualizar $\mathrm{y}$ transparentar a todos los integrantes de las organizaciones presentes los insumos a ser tomados en cuenta en el informe por parte del asistente técnico. No se realizaron metodologías de observación en campo, mediciones, análisis SIG u otras metodologías de monitoreo para elaborar los informes semestrales.

\section{Discusión}

En la literatura sobre monitoreo comunitario y participativo se menciona que la responsabilidad de dicho monitoreo puede estar con los usuarios de los recursos naturales, asistentes técnicos, entes del Estado, o investigadores de universidades (Abbot y Guijt ,1998; Evans, 2008). En el marco de los AUSCM, la organización de pescadores se compromete a realizar los informes semestrales, por lo que por normativa la responsabilidad cae en manos de las organizaciones, siendo según Evans (2008), un monitoreo comunitario y participativo con un nivel de participación con empoderamiento. En la práctica, la elaboración de los informes semestrales se lo realiza con apoyo de profesionales, como es el caso de las 10 organizaciones a las cuales, docentes y estudiantes de la UTPL/GIZ, apoyaron en recolección de la información (por medio de talleres, encuestas, entrevistas y revisión de documentación) y la plasmaron en el informe. Según la categorización de Evans, esta forma de trabajo puede considerarse como participación consultiva. Cabe anotar que según el estudio de efectividad de manejo realizado en el Oro por la UTPL (2017), con la debida capacitación y una simplificación del formato de los informes semestrales, los líderes de las organizaciones podrían obtener las destrezas y conocimiento para encargarse de elaborar los informes semestrales.

No se encontraron estudios sobre el manejo o monitoreo comunitario y participativo de los manglares en escala nacional o mundial, CIFOR en una investigación sobre gobernanza de manglares, destaca la experiencia de gobernanza participativa con AUSCM en Ecuador, y en cuanto al manejo comunitario de manglares recomienda una investigación comparativa a nivel mundial para poder sacar lecciones aprendidas para tomadores de decisión (Rotich, 2016).

De la literatura sobre el monitoreo elaborada por Abbot y Guijt (1998), UNHCR (2008), Stelzenmuller (2013), Evans (2018), Fröde y Masara (2007) se extrajeron los siguientes temas: 1) estado de los recursos naturales y su ecosistema 2) estado de especies útiles 3) amenazas a los recursos naturales 4) acciones de manejo 5) cumplimiento de la normativa 6) análisis de efectividad de un proyecto. Aunque el énfasis de los informes semestrales de los AUSCM está en informar al MAE el cumplimiento de los 
programas y acciones del Plan de Manejo, contienen información sobre las demás categorías del estudio (uso sustentable de recursos bioacuáticos, amenazas y anomalías, desarrollo organizacional, cumplimiento de normativa, etc.). Con un ajuste en el formato de los informes semestrales y unas capacitaciones en técnicas sencillas y económicas de monitoreo se podría potenciar el monitoreo en estos temas adicionales para así llegar a un monitoreo participativo y comunitario más completo de las áreas de custodia y generar información útil para la toma de decisiones en favor de la conservación del manglar.

Los manuales sobre metodologías de monitoreo participativo presentan una serie de técnicas para dicho monitoreo (Bautista et al., 2004; Gallego, 2017). Las 10 organizaciones objeto de este estudio y la UTPL-GIZ han utilizado principalmente talleres y la revisión de evidencias (físicas y digitales), pero existen otras metodologías que podrían usarse para recolectar datos que hasta la fecha no se han incluido en los informes semestrales. El MAE se encuentra trabajando con actores de apoyo como la UTPL, GIZ, Fundación Heifer y Conservación Internacional (CI) para elaborar metodologías de monitoreo participativo con base en fichas y aplicaciones móviles (ManglarApp, InfoManglar).

Del análisis de la normativa sobre los AUSCM, la literatura sobre Monitoreo Comunitario y Monitoreo Participativo y la experiencia en la elaboración de informes semestrales con 10 organizaciones se mostró que la información recolectada y la metodología usada se enmarcan en el concepto conocido a nivel mundial como el "Monitoreo participativo y comunitario de los recursos naturales".

Los informes recogen principalmente información sobre el cumplimiento o no de las actividades de sus Planes de Manejo, y no abordan de manera detallada los impactos de la gestión en favor de la conservación del manglar, la reducción de las amenazas, o, en general, el estado en el cual se encuentran los manglares. Se recomienda al MAE y las organizaciones con AUSCM realizar un ajuste al formato de los informes semestrales y realizar unas capacitaciones en técnicas sencillas y económicas de monitoreo para así potenciar el monitoreo en generar un panorama más completo de información sobre los manglares.

El Plan de Acción Nacional para la Conservación de los Manglares del Ecuador Continental indica la necesidad de contar con más información sobre los manglares del país. Hasta ahora, los informes semestrales han sido poco aprovechados como fuente de información, pero tienen el potencial para ser utilizados por investigadores, técnicos y tomadores de decisión en cuanto a políticas, normativas y programas. Se recomienda analizar medidas para facilitar la organización de la información recolectada y el acceso a la misma. establecer alianzas con universidades para su apoyo en la interpretación de los datos de estos informes y presentar las conclusiones a tomadores de decisión. 
Aunque la responsabilidad de realizar los informes semestrales es de los usuarios del manglar, la experiencia práctica ha demostrado que las organizaciones dependen, en mayor o menor medida, del apoyo de la entidad de asistencia técnica, el MAE o un consultor. Se recomienda incluir en el marco normativo una propuesta de metodología de trabajo del ente de apoyo, en el cual se presenten las técnicas participativas de monitoreo y reporte de la información a seguir cuando se elaboran los informes.

Fuentes de financiamiento: Cooperación Técnica Alemana y Universidad Técnica Particular de Loja.

\section{Referencias}

Abbot, J y Guijt, I. (1998) Changing views on change: participatory approaches to monitoring the environment. SARL Discussion Paper No 2. July 1998. Disponible en: https://pubs.iied.org/ 6140IIED/

Acuerdo Ministerial No 129. (2010) Registro Oficial No. 283. Quito: Ministerio del Ambiente. 21 de septiembre del 2010. Disponible en http://gobec.gobiernoelectronico.gob. ec/sites/default/files/regulations/2018-09/ Documento_Procedimiento-Acuerdo-Us o-Sustentable-Custodia-Manglares.pdf

Acuerdo Ministerial No 144. (2011) Registro oficial No 568, Quito: Ministerio del Ambiente. http://cpps.dyndns.info/cpps-docs-web/pl anaccion/docs2013/manglares/Acuerdo $\%$ 20144.pdf

Alongi, DM. (2002) Present state and future of the world's mangrove forests. Environmental Conservation 29(3). Cambridge University Press: 331-349. DOI: 10.1017/S037689 2902000231.
Bandaranayake, WM. (1998) Traditional and medicinal uses of mangroves. Mangroves and Salt Marshes 2(3): 133-148. DOI: 10.1023/A:1009988607044.

Bautista, F etal. (2004). Técnicas de muestreo para manejadores de recursos naturales. Disponible en: https://www.researchgate. net/publication/236954619_Tecnicas_de _muestreo_para_manejadores_de_recurs os_naturales

Capote-Fuentes, R. y R. Levins. (2006) Modelación matemática cualitativa como apoyo a la dirección de la gestión ambiental. Tercer Seminario Bienal Internacional acerca de las Implicaciones Filosóficas, Epistemológicas y Metodológicas de la Teoría de la Complejidad. Habana, Cuba.

Carvajal R. y X. Santillán. (2019). Plan de Acción Nacional para la Conservación de los Manglares del Ecuador Continental. Ministerio del Ambiente de Ecuador, Conservación Internacional Ecuador, Organización de las Naciones Unidas para la Educación, la Ciencia y la Cultura (UNESCO) y la Comisión Permanente del Pacífico Sur (CPPS). Proyecto Conservación de Manglar en el Pacífico Este Tropical. Guayaquil, Ecuador. Disponible en: http://conservation.org.ec/ wp-content/uploads/2019/07/PAN-Mangl ares-Ecuador.pdf

Código Orgánico del Ambiente (COA). (2017). Registro Oficial Suplemento No. 983. Quito: Asamblea Nacional del Ecuador. 12 de abril del 2017. Disponible en: http://www.competencias.gob.ec/wp-cont ent/uploads/2017/06/05NOR2017-COA. pdf

CONABIO. (2005) Sistema de Monitoreo de Manglares de México. Disponible en: https://www.biodiversidad.gob.mx/ecosis temas/manglares2013/smmm.html

Evans, K. (2008) Monitoreo Participativo para el manejo forestal en el trópico: una revisión 
de herramientas, conceptos y lecciones aprendidas/Kristen Evans y Manuel R. Guariguata. Bogor, Indonesia. Disponible en: https://www.researchgate.net/publica tion/235936033_Participatory_Monitorin $\mathrm{g}$ in tropical_forest management_a revie w_of_tools_concepts_and_lessons_learned

Evans, K. (2016). Guía práctica para el monitoreo participativo de gobernanza. Disponible en:https://www.researchgate.net/publicati on/315708338_Guia_para_el_monitoreo participativo_de_la_gobernanza. Bogor, Indonesia: $\overline{\mathrm{CIFOR}}$ Centro para la Investigación Forestal Internacional (CIFOR), 2008.

Fröde, A. and Masara, C. (2007). Community-based ecological monitoring. Manual for practitioners, SAFIRE. Harare, Zimbabwe. Disponible en: http://www.fao.org/ forestry/14700-0271f3fb3f50174269227f d97906437a9.pdf

Gallego, I. (2017) El enfoque del Monitoreo y la evaluación participativo (MEP): Bateria de herramientas metodológicas. Disponible en: df02dd5938850061602215b3312200f c. pdf

GIZ. (2013) Deutsche Gesellschaft für Internationale Zusammenarbeit (GIZ) GmbH. 2013. Herramientas de Gestión en Áreas Protegidas en Ecuador: Experiencia Práctica en el Parque Nacional SumacoNapo Galeras. Serie sistematización No. 14 Quito.

INVEMAR. (2012). Monitoreo de Manglares de la Ciénaga grande de Santa Marta. Bogotá, Colombia. http://cinto.invemar. org.co/alfresco/d/d/workspace/SpacesSto re/e58fa257-7f6f-47ab-8a02-87e17a4eaa 88/Monitoreo\%20de\%20Manglares $\% 20$ Ci\%C3\%A9naga\%20Grande\%20de\%20 Santa\%20Marta?ticket=TICKET_dc95c1 2a782c3b28aceff1d1c6758c8edfc36aff

López-Rodríguez, FV. (2018) Mangrove Concessions: An Innovative Strategy for Community
Mangrove Conservation in Ecuador. In: Makowski C and Finkl CW (eds) Threats to Mangrove Forests: Hazards, Vulnerability, and Management. Cham: Springer International Publishing, pp. 557-578. doi: 10.1007/978-3-319-730165_25

Ministerio del Ambiente del Ecuador. (2019). Plan de Acción Nacional para la Conservación de los Manglares del Ecuador. Guayaquil, Ecuador.

McIvor A, et al. (2015) Chapter 14 - Mangroves, Tropical Cyclones, and Coastal Hazard Risk Reduction. In: Shroder JF, Ellis JT, and Sherman DJ (eds) Coastal and Marine Hazards, Risks, and Disasters. Boston: Elsevier, pp. 403-429. doi.org/10.1016/B978-0-12-396483-0.00 014-5.

Menéndez, J. (2012) Programa de Monitoreo de Manglares de Cuba. Habana, Cuba. Disponible en: https://www.undp.org/ content/dam/cuba/docs/Medio\%20Ambi ente $\% 20 y \% 20$ Energ $\%$ c3\%ada/Publicaci ones/5.\%20Reporte $\% 20$ Programa $\% 20 \mathrm{de}$ $\% 20$ Monitoreo $\% 20$ Manglar $\% 20$ final $2 \%$ 20prueba.pdf

Menéndez, L. (2013). El ecosistema de manglar en el archipiélago cubano: bases para su gestión [en línea]. España: Universitat d'Alacant. Disponible en: https://ialnet. unirioja.es/servlet/tesis? codigo $=59555$.

Menéndez, L. (2000): Bases Ecológicas para la Restauración de Manglares en Áreas Seleccionadas del Archipiélago Cubano y su relación con los Cambios Climáticos Globales. Habana, Cuba.

PNUD-IAVH. (2017). Propuesta de monitoreo comunitario a la Restauración. Instituto Alexander von Humboldt. Bogotá. Colombia Disponible en: http://reposito ry.humboldt.org.co/bitstream/ handle/20.500.11761/35300/P\%204.3 \%20Monitoreo\%20Comunitario.pdf?seq 
uence $=2 \&$ is Allowed $=\mathrm{y}$

Rotich, B., Mwangi, E. \& Lawry, S. (2016). Where land meets the sea: a global review of the governance and tenure dimensions of coastal mangrove forests. Bogor, Indonesia: CIFOR; Washington, DC: USAID Tenure and Global Climate Change Program.

Stelzenmüller, V. et al. (2013) Monitoring and evaluation of spatially managed areas: A generic framework for implementation of ecosystem based marine management and its application. Marine Policy Vol 37, pp 149-164. doi.org/10.1016/j.marpol.2012. 04.012

UTPL. (2017). Evaluación de efectividad de manejo de los acuerdos de uso sustentable y custodia del manglar en la provincia de El Oro (Documento digital). SGMC, CI-Ecuador, HIVOS, GEF y FAO. Loja. Ecuador. 